\title{
SULL'OMOGENEITÀ E SULLO STATO DI EQUILIBRIO DEL MANTELLO INTERNO DELLA TERRA
}

\author{
P. E. VAlLe
}

Introduzione. - In una nota precedente $\left(^{1}\right)$ è stato posto in evidenza che le velocità delle onde elastiche di agitazione termica di un solido isotropo, omogeneo, di tipo monoatomico, sono date dalle relazioni

$$
v_{\mathrm{m}}=v_{\mathrm{om}}\left(\frac{\rho_{\mathrm{o}}}{\rho}\right)^{1 / 3} e^{\gamma_{\mathrm{om}}\left(1-\frac{\varrho_{\mathrm{o}}}{\mathrm{e}}\right)} \quad(m=l, t)
$$

nelle quali $\rho$ è la densità, Yom un parametro e gli indici $l$ e $t$ si riferiscono alle onde longitudinali e trasversali rispettivamente.

Queste velocità sono determinate dai moduli valutati ad energie termiche costanti, ma se la temperatura del solido è superiore alle temperature caratteristiche, coincidono con le velocità isoterme.

In questa nota si studiano alcuni particolari dell'andamento di tali velocità in uno strato omogeneo, in equilibrio idrostatico, in relazione al gradiente di temperatura e nell'ipotesi che la temperatura stessa sia superiore alle temperature caratteristiche.

Come applicazione viene tentata una verifica, per il mantello interno della Terra, delle ipotesi su cui poggia il calcolo del modello $A$ di K. E. Bullen.

Si premettono alcune considerazioni sulle trasformazioni adiabatiche reversibili.

Trasformazioni adiabatiche. - Se la temperatura è superiore alle temperature caratteristiche, l'entropia di un solido può essere espressa, con sufficiente approssimazione, dalla relazione (1)

$$
S=-\frac{R}{A} \Sigma \log \frac{\theta_{\mathrm{m}}}{T} \quad(m=l, t, t)
$$

nella quale $R$ è la costante dei gas, 4 il peso atomico, $\theta_{\mathrm{m}}$ sono le temperature caratteristiche e $T$ la temperatura assoluta. 
Se si indica con lindice zero un certo stato iniziale si ha dalla [2]

$$
\frac{A}{R}\left(S-S_{0}\right)-\log \frac{T^{3} \theta_{01} \theta_{0}}{T_{0}{ }^{3} \theta_{1} \theta_{i}^{2}}
$$

o anche, tenendo presente che le temperature caratleristiche sono proporzionali alle lrequenze massime di vibrazione,

$$
\frac{A}{R}\left(S-S_{0}\right)=\log \frac{T^{3} \nu_{01} \nu^{2}{ }_{\ldots 1}}{T_{0}{ }^{3} \nu_{1} \nu_{1}^{2}}
$$

Ora nella nota wià cilata ${ }^{1}{ }^{1}$ è stato dimostrato che

$$
v_{\mathrm{m}}=v_{\mathrm{m}} e^{i_{\mathrm{om}}\left(1-\frac{Q_{0}}{g}\right)}
$$

e quindi risulta

$$
T=T_{0} e^{\ddot{O}_{0}\left(1-\frac{0_{0}}{0}\right) \div \frac{\left(\dot{c}-s_{0}\right)}{r_{v}}}
$$

nella quale $\gamma_{0}$ è il parametro di Grüneisen, dato da

$$
\Upsilon_{0}=\frac{Y_{01}+2 \gamma_{o t}}{3}
$$

e $c_{r}$ è il valore classico del calore specifico a volume costante, ossia

$$
c=\frac{3 R}{A}
$$

Se si considera una trasformazione adiabalica reversibile che porti il solido dalla densità $p_{0}$ alla densita $p, 1 a[4]$ diviene

$$
T=T_{a} e^{i 0}\left(1-\frac{00}{0}\right)
$$

Questa relazione si trova già accennala in precedenti lavori dell'Autore e può essere scritta anche nella forma

$$
\frac{T^{3}}{\rho v_{1} v_{1}{ }^{3}}=\text { costante }
$$


Si può vedere facilmente che se la temperatura iniziale $T_{0}$ soddisfa la condizione

$$
T_{0}>\theta_{\mathrm{mm}} e^{\gamma_{\mathrm{om}}-\gamma_{0}}
$$

una compressione adiabatica mantiene la temperatura sempre al disopra delle temperature caratteristiche.

Gradienti di temperatura. -- Si consideri uno strato omogeneo, isotropo, nel quale la variazione della velocità $v_{\mathrm{m}}$ con la profondità $h$ è determinata soltanto dalla variazione della densita $p$ e non dai valori iniziali dei parametri che compaiono nella [1]. Si avà

$$
\frac{d v_{m}}{d h}=\frac{\partial v_{m}}{\partial \rho} \frac{d \rho}{d h}
$$

La densità p può essere pensata funzione della temperatura $T$ e della pressione If attraverso l'equazione di stato, e quindi

$$
\frac{d v_{\mathrm{m}}}{d h}=\frac{\partial v_{\mathrm{m}}}{\partial \rho}\left(\frac{\rho}{K_{\mathrm{T}}} \frac{d p}{d h}-\approx \frac{d T}{d h}\right)
$$

nella quale $K_{\text {. }}$ ì il modulo di compressibilita isoterma e $\alpha$ la dilatazione termica.

Se l'equilibrio is idrostatico la [11] si scrive

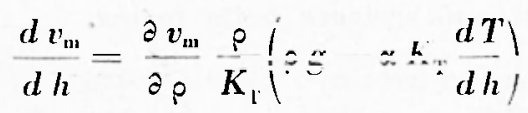

dove $g$ i il campo gravitazionale.

Conviene introdurne il gradiente di temperatura velocita rimane costante al variare di $h$ e che verri chiamato gradiente critico. Si avri

$$
\tau_{c}=\frac{\rho g}{\alpha \cdot K_{\mathbf{T}}}
$$

o anche, ricordando che $\alpha K_{\mathrm{t}}=\gamma_{\mathrm{p}} c_{\mathrm{v}}=\gamma_{\mathrm{o}} \rho_{\mathrm{o}} \mathrm{e}_{\mathrm{v}}$,

$$
\tau_{\mathrm{c}}=\frac{g}{\gamma_{\mathrm{o}} c_{\mathrm{v}}} \frac{\rho}{\rho_{\mathrm{o}}}
$$


Indicando con $\tau$ il gradiente attuale $d T / d h$, dato che dalla [I] risulta

$$
\frac{\partial \log v_{\mathrm{m}}}{\partial \rho}=\frac{1}{\rho}\left(\gamma_{\mathbf{n}}-\frac{1}{3}\right)
$$

perche $\gamma_{m} \rho=\gamma_{o m} \rho_{0}$, l'equazione [12] diventa

$$
\frac{d \ddot{\nu}_{\mathrm{m}}}{d h}=\frac{\ddot{v}_{\mathrm{m}}}{K_{\mathrm{T}}} \rho g\left(\gamma_{\mathrm{m}}-\frac{1}{3}\right)\left(1-\frac{\tau}{\tau_{\mathrm{e}}}\right)
$$

Questa relazione può essere ulteriormente trasformata introducendo il gradiente adiabatico

$$
\tau_{\mathrm{s}}=\frac{T \alpha g}{c_{\mathrm{p}}}
$$

Con qualche passaggio dalla [13] e dalla [16] risulta

$$
\frac{\tau_{\mathrm{s}}}{\tau_{\mathrm{c}}}=\frac{\alpha{ }^{\mathrm{T}} \mathrm{T}}{1+\alpha \gamma \mathrm{T}}
$$

Dato che in grenerale $\alpha>0, \tau_{c}$ è sempre matriore di $\tau_{s}$.

Ora indicando con $K_{s}$ il modulo di compressibilita adiahatica si ha

$$
\frac{K_{i}}{K_{\mathrm{T}}}=\frac{1}{1-\frac{\tau_{\mathrm{s}}}{\tau_{\mathrm{c}}}}
$$

e quindi la [15] si può scrivere nella forma

$$
\frac{d v_{\mathrm{m}}}{d h}=v_{\mathrm{m}} \frac{\rho g}{K_{\mathrm{S}}}\left(\gamma_{\mathrm{m}}-\frac{1}{3}\right)^{\frac{1-\frac{\tau}{\tau_{\mathrm{c}}}}{1--\frac{\tau_{\mathrm{s}}}{\tau_{\mathrm{c}}}}}
$$

oppure

$$
A_{\mathrm{m}} \boldsymbol{v}_{\mathrm{m}} \frac{\boldsymbol{d} \boldsymbol{v}_{\mathrm{m}}}{d h}=g\left(\gamma_{\mathrm{m}}-\frac{1}{3}\right) \frac{1-\frac{\tau}{\tau_{\mathrm{c}}}}{1-\frac{\tau_{\mathrm{s}}}{\tau_{\mathrm{c}}}}
$$

dove si ̀̀ posto

$$
A_{\mathrm{m}}=\frac{\left(v_{1}^{2}\right)_{5}-\frac{4}{3} v_{1}^{2}}{v_{\mathrm{m}}^{2}}
$$


Si osservi che la [20] mostra che le velocità isoterme sono stazionarie al centro della Terra perché ivi è $g=0$.

Effettivamente la velocità delle onde sismiche lonsitudinali, la quale però $\grave{e}$ la velocità adiabatica, presenta un andamento del grenere verso il centro della Terra.

Posto ora

$$
R_{\mathrm{m}}=\frac{A_{\mathrm{m}} v_{\mathrm{m}}}{g\left(\gamma_{\mathrm{m}}-\frac{1}{3}\right)} \frac{d v_{\mathrm{m}}}{d h}
$$

nell'ipotesi che la densità $p$ si mantenga sempre minore di $3 \gamma_{0 m} \rho_{0}$, ossia nell'ipotesi che risulti $\gamma_{m}>1 / 3$, ricordando che $\tau_{s}<\tau_{c}$, si hanno i casi notevoli indicati nella Tabella $I$.

TABELLA I

\begin{tabular}{|c|c|}
\hline Valore di $R m$ & Valore di $\tau$ \\
\hline$R m<0$ & $\tau>\tau_{c}$ \\
$R m=-0$ & $\tau=\tau_{\mathrm{s}}$ \\
$R m<1$ & $\tau>\tau_{\mathrm{s}}$ \\
$R m=1$ & $\tau=\tau_{\mathrm{s}}$ \\
$R m>1$ & $\tau<\tau_{\mathrm{s}}$
\end{tabular}

Può essere interessante talvolta considerare anche il gradicnte di temperatura $\tau_{0}$, necessario perché si formi una zona d'ombra per la propagazione delle onde elastiche.

Com i noto la formazione della zona dombra si ha quando

$$
\frac{1}{v_{\mathrm{m}}} \frac{d v_{\mathrm{m}}}{d h}=-\frac{1}{r_{\mathrm{o}}-h}
$$

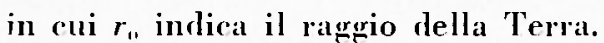

Introducendo questa condizione nella $[20]$ si ha

$$
-\frac{A_{\mathrm{m}} v_{\mathrm{m}}^{2}}{r_{\mathrm{o}}-h}=g\left(\gamma_{\mathrm{m}}-\frac{1}{3}\right)^{1-\frac{\tau_{\mathrm{o}}}{\tau_{\mathrm{c}}}}
$$


mediante la quale si può valutare $\tau_{0}$. Questo gradiente, nell'ipotesi $\gamma_{m}>1 / 3$ ì superiore al gradiente critico.

Tentativo di verifica delle ipotesi sulle quali is stato costruito il Modello A di K. E. Bullen per il mantello interno della Terra. - I.e più recenti discussioni sulle proprietà elastiche, temperatura c composizione del mantello interno della Terra, sono probabilmente quelle che si sono sviluppate sulla base dei lavori presentati da F. Birch (?) e J. Verhoogen $\left(^{3}\right)$ alla $34^{a}$ riumione annuale dell'American Geophysical Union.

Peraltro, allo stato delle attuali conoscenze, sembra opportuno controllare se le note ipotesi sulle quali ̀े stato costruito il modello $A$ di K. E. Bullen sono verificate.

Un tale controllo può essere tentato mediante la relazione [20] del paragrafo precedente, prendendo in considerazione le onde sismiche di tipo $S$, perché la velocitì isoterma di queste onde coincide con la velocitì adialoatica.

L'applicabilità della teoria dei solidi all’interno della Terra è stata discussa in precedenti lavori $\left(^{(1-5)}\right.$.

Se lo strato $D$ è omogeneo, in equilibrio idrostatico e adiabatico dovrà risultare

$$
\mathbf{R}_{\mathbf{t}}=1
$$

ossia

$$
A_{\mathrm{t}} v_{\mathrm{t}} \frac{d v_{\mathrm{t}}}{d h}=g\left(\gamma_{\mathrm{t}}-\frac{1}{3}\right)
$$

Il primo membro della precedente relazione puó essere diret1amente calcolato dai valori delle velociti delle onde sismiche, mentre il secondo membro può essere valutato soltanto in hase ad un modello perché contiene $g$ e $\gamma$.

Nella Tabella II sono contenute le velociti delle onde sismiche calcolate da $H$. Jeffreys $\approx$ e le grandezze relative al modello $A$ di K. E. Bullen $(i-s)$.

Dato che si presenta qualche difficoltà per il calcolo di $d v_{v} / d h$, la velocita $v_{t} i$ stata approssimata, mediante il metodo dei minimi quadrati, con una curva di tipo parabolico. 


\section{TABELLA II}

\begin{tabular}{|c|c|c|c|c|}
\hline $\begin{array}{c}h \\
\text { kn }\end{array}$ & $\begin{array}{c}\left(v_{1}\right)_{\mathrm{s}} \\
\mathrm{km} / \mathrm{sec}\end{array}$ & $\begin{array}{c}\mathrm{t}_{1} \\
\mathrm{~km} / \mathrm{sec}\end{array}$ & $\begin{array}{c}\text { g! } \\
\operatorname{com}^{3}\end{array}$ & $\begin{array}{c}g \\
\operatorname{con} / \sec ^{2}\end{array}$ \\
\hline $\begin{array}{l}1000 \\
1200 \\
1400 \\
1600 \\
1800 \\
2000 \\
2200 \\
2400 \\
2600 \\
2800\end{array}$ & $\begin{array}{l}11,42 \\
11, ?] \\
11,99 \\
12,26 \\
12,53 \\
12,79 \\
13,03 \\
13,27 \\
13,50 \\
13,61\end{array}$ & $\begin{array}{l}6,36 \\
6,50 \\
6,62 \\
6,73 \\
6,83 \\
6,93 \\
7,02 \\
7,12 \\
7,21 \\
7.30\end{array}$ & $\begin{array}{l}4,68 \\
1,80 \\
4,91 \\
5,03 \\
5,13 \\
5,24 \\
5,34 \\
5,44 \\
5.54 \\
5,63\end{array}$ & $\begin{array}{r}995 \\
991 \\
988 \\
986 \\
985 \\
985 \\
990 \\
993 \\
1009 \\
1026\end{array}$ \\
\hline
\end{tabular}

Con i valori della Tabella II si è ottenuto:

$$
\begin{aligned}
\frac{v_{1}}{v_{11}}-1 & =(0,101747 \ldots 0,001862) \mathrm{h}^{\prime} \\
& -(0,011346+0,001270) \mathrm{h}^{\prime \prime}
\end{aligned}
$$

dove

$$
\mathrm{h}^{\prime}=10^{-i}(\mathrm{~h}-1000)
$$

e con lindice $l$ si $\grave{e}$ indicato il valore della velocità alla sommità dello strato $D$, ossia alla profonditi di $1000 \mathrm{~km}$.

La derivata risulta quindi

$$
\begin{gathered}
\frac{d v_{\mathrm{t}}}{d h}=6,3610^{3}[(0,101747 \div 0,001862\rfloor- \\
\left.-0,022692 \div 0,002510) \mathrm{~h}^{\prime}\right]
\end{gathered}
$$

Sono stati ritenuti esatti i valori delle velociti di Jeffreys. Lerrore medio preso in considerazione è soltanto l'errore medio della derivata. In questo modo è stato calcolato il primo membro della relazione [26] e i risultati sono contenuti nella seconda colonna della Taluella III. 
Tabella III

\begin{tabular}{|c|c|c|c|}
\hline $\begin{array}{c}\mathrm{l} \\
\mathrm{km}\end{array}$ & $\begin{array}{l}A_{t}, \frac{d}{d} \frac{d}{d h} \\
\mathrm{~cm} / \mathrm{sec}^{2}\end{array}$ & $\begin{array}{c}g\left(\gamma_{t}-1 / 3\right) \\
(\mathrm{m} / \mathrm{sec}=\end{array}$ & $R_{t}$ \\
\hline $\begin{array}{l}1000 \\
1200 \\
1400 \\
1600 \\
1800 \\
2000 \\
2200 \\
2400 \\
2600 \\
2800\end{array}$ & $\begin{array}{l}778 \div 14 \\
768 \div 15 \\
760 \div 17 \\
749 \div 20 \\
738 \div 24 \\
722 \div 29 \\
703-34 \\
678-39 \\
652 \div 4 \\
610 \div 49\end{array}$ & $\begin{array}{l}831-4 \\
799+4 \\
771+4 \\
714+4 \\
722-3 \\
701+3 \\
684+3 \\
671-3 \\
660+3 \\
655 \div 3\end{array}$ & $\begin{array}{c}0.936 \pm 0,017 \\
0,961 \pm 0,019 \\
0,986 \pm 0,023 \\
1,007 \pm 0,027 \\
1,022 \pm 0,033 \\
1,030 \pm 0,042 \\
1,028 \pm 0,050 \\
1,010 \pm 0,058 \\
0,988 \pm 0,067 \\
0,931 \pm 0,075 \\
\text { Media } 0,975 \pm 0,011 \\
\text { (ponder:at) }\end{array}$ \\
\hline
\end{tabular}

In un precedente lavoro $\left(^{n}\right)$ è stato dedotto dalle velocità di Jeffreys e dalle densità del modello A di Bullen, il valore del parametro $\gamma_{\text {t }}$ alla sommita dello strato $D$, cioè alla profonditi di $1000 \mathrm{~km}$. Tale valore, considerato con quattro cifre decimali, i il seguente:

$$
\gamma_{11}=1,1689+0,0039
$$

Ricordando che

$$
\gamma_{1}=\gamma_{12} \frac{\rho_{1}}{\rho}
$$

si possono calcolare facilmente $i$ valori di $\gamma$ alle varie profonditi, mediante i valori della densiti della Tabella II.

La terza colonna della Tabella III contiene il prodotto $g\left(\gamma_{t}-1 / 3\right)$. L'errore medio indicato è soltanto quello the deriva dall'errore medio di $\gamma:$

Lultima colonna della Tabella IIl contiene infine il rapporto $R_{t}$. il cui errore medio indicato è stato calcolato approssimativamente con gli errori medi attribuiti al dividendo e al divisore. Questo rapporto è riportato in funzione della profonditi nella fig. 1 .

Prima di trarre qualche conclusione dai risultati numerici contenuti nella Tabclla HI, ̀̀ necessario eseguire una stima del gradiente critico -... 
Se si suppone, a titolo di orientamento, che il peso atomico medio dei materiali che costituiscono lo strato $D$ sia uguale a 20 , Ia relazione

$$
\tau_{c}=\frac{c_{v} \gamma_{1}}{r} \frac{\rho}{p_{1}}
$$

nella quale l'indice 1 si riferisce alla sommità dello strato, consente di calcolare $\tau_{\text {c, }}$ in funzione della profondità, con i dati della Ta-

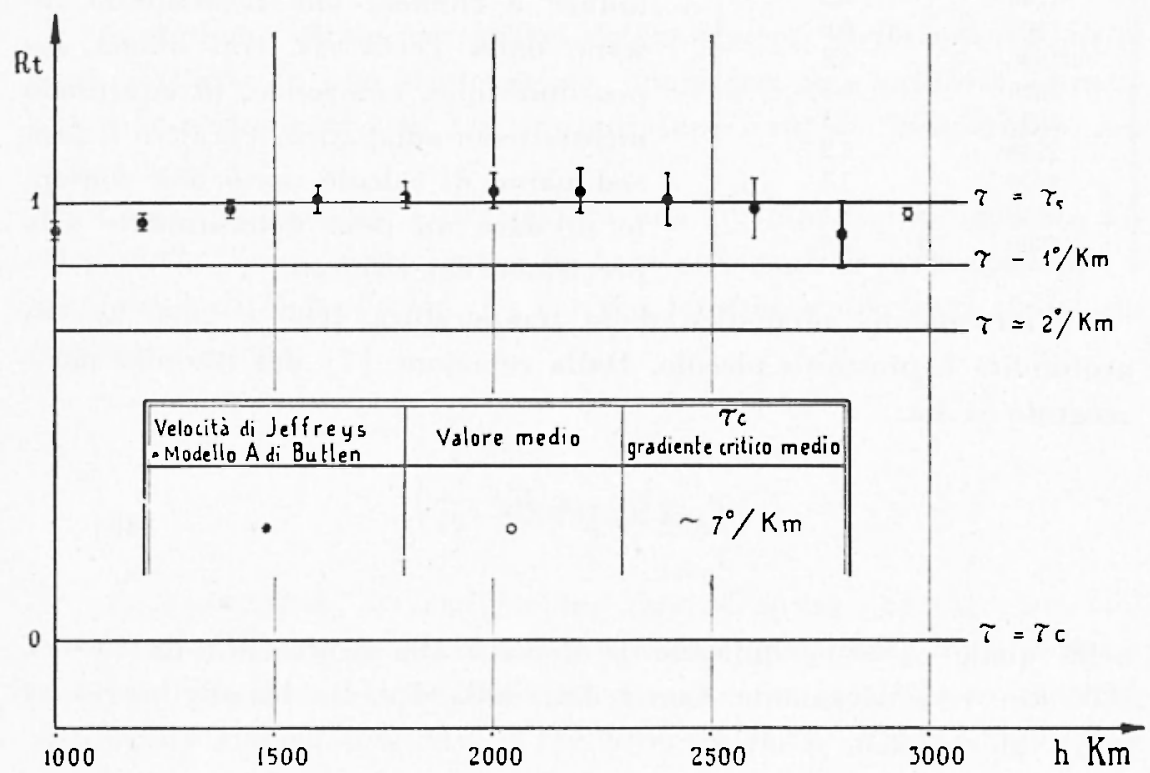

Fig. 1

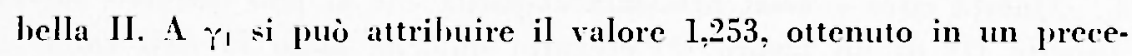
dente lavoro ( $\%$.

Lal Tabella IV mostra che - i compreso tra 6,4 gradi $\mathrm{km}$ e 7,9 gradi $\mathrm{km}$. In media $\tau$. si può assumere pari a $\tau$ gradi $\mathrm{km}$. Ora, trascurando $\tau_{: s}$ rispetto a $\tau_{\text {... }}$ si ha

$$
R_{\mathrm{t}}=1-\frac{\tau}{\tau_{\mathrm{c}}}
$$


Se si attrihuiscono a $=\mathrm{i}$ valori $1 ; 1,5 ; 2$ gradi/km, $R_{1}$ risulta pari a circa $0.86: 0,79 ; 0.71$ rispeltivamente.

\section{Tabella IV}

\begin{tabular}{c|c|}
\hline$h$ & $\tau_{c}$ \\
$\mathrm{~km}$ & gridi $/ \mathbf{k m}$ \\
& \\
\hline & \\
1000 & 6,4 \\
1200 & 6,5 \\
1400 & 6,6 \\
1600 & 6,8 \\
1800 & 6,9 \\
2000 & 7,1 \\
2200 & 7,2 \\
$2+00$ & 7,4 \\
2600 & 7,6 \\
2800 & 7,9
\end{tabular}

Queste cifre, pur essendo puramente indicative, danno una idea della diminuzione del rapporto $R_{\mathrm{t}}$ con l'aumentare di $\tau$.

Dall'esame della 'labella 111 risulta in primo luogo che il gradiente attuale $\tau$ è molto minore del gradiente

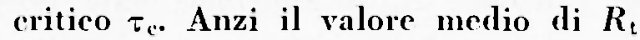
induce a ritenere che il mantello interno della Terra sia, con buona approssimazione, omogeneo, in equilibrio idrostatico e adiabatico. Peraltro il procedimento di calcolo usato non consente di dare un peso determinante alla precisione dei risultati.

L'incremento adiabatico della temperatura 1000 e $2800 \mathrm{~km}$ di profondità è piuttosto piccolo. Dalla relazione [7] del secondo paragragrafo si ha

$$
\frac{T_{2800}}{T_{1000}}=e^{\gamma_{1}\left(1-\frac{\varrho_{1}}{\varrho_{2}}\right)}
$$

nella quale $\rho_{1}$ e $\rho_{2}$ indicano la densità alla profonditi di 1000 e $2800 \mathrm{~km}$. rispettivamente. Con i dati della Tabella II, attribuendo a $\because 1$ il valore 1.253 , si ottiene

$$
-{\frac{T}{T_{1000}}}^{000}=1.235
$$

Questa cifra i assai prossima a quella che si può dedurre dalla Tabella III di un lavoro (9) eseguito qualche anno fa dall'Autore. Da detta Tabella risulta

$$
\frac{T_{\mathrm{sou}}}{T_{\mathrm{1100}}}=\frac{1,62_{\mathrm{s}}}{1,31}=1,24
$$

Si può quindi concludere che se l'equilibrio del mantello interno della Terra è prossimo all'equilibrio adiahatico, come sembra mostrare 
la presente ricerca, il gradiente di temperatura nel suo interno è piccolo, probabilmente pari ad alcuni decimi di grado per $\mathrm{km}$, anche ammettendo che la temperatura a $1000 \mathrm{~km}$ di profondità sia pari a qualche migliaio di gradi.

$$
\text { Roma -- Istituto Nazionale di Geofisica - Marzo } 1954 .
$$

\section{RIASSUNTO}

Si studiano alcuni particolari dellandamento della velocità delle onde elastiche in uno strato solido, omogeneo, in equilibrio idrostatico. che si trovi ad una temperatura superiore alle temperature caratteristiche.

Come applicazione viene tentata una verifica, per il mantello interno della Terra, delle ipotesi in base alle quali è stato costruito il modello A di K E. Bullen. La verifica fornisce, entro certi limiti, un risultato positivo.

\section{SUMMARY}

An equation is derived for the rate of change of the isotermal velocities of elastic waves, in a homogeneous gravitating layer with an arbitrary gradient of temperature. An attempt is made 10 test the uniformity and adiabaticity of the layer D of the Earth's muntle on the basis of this equation.

\section{BIBLIOGRAFIA}

(1) Valde P. F... Una equazione di stato per i solidi. Amnali di Geofisiea, 6, 183-197 (1953).

(2) Bunch F. The Earth's Mamtle - Elasticity and Constimtion. Trans. Am. Geoplys. Union, 35, 79-85 (1054).

(ib) Venhoogen J., Petrological evidence on temperature distribution in the mante of the Earth. Trans. Am. Geoplyys. Union, 35, 85-92 (1954).

(4) Buncin F., Elasticily and constitution of Earth's interior. J. Geophys. Res.. $57,227.286$ (1952). 
(5) Valle P. E., Sulle proprietì elastiche del mantello della Terra. Annuli di Geofisica, 6, 373-380 (1953).

(6) Jefrreys H., The time of $P, S$ and SKS and the velocieties of $P$ and $S$. Geophys. Suppl. 4, 498-533 (1939).

(i) Bulcex K. E., The problen of the Earth's density variations. Bull. Seism. Soc. Am., 32, 19-29 (1942).

(8) BulleN K. E, Introduction to the theory of Seismology. Cambridge, University Press (1947).

(9) Valle P. E., Sul gradiente adiabatico di temperatura nellinterno della Terra. Annali di Geofisica, 5, $41-53$ (1952). 